\author{
மரபுவழி அடையாளமும் வாழும் நாட்டின் அடையாளமும் \\ எழுத்தாளர் கே. எஸ். மணியம் படைப்புகளில் அடையாளத் தேடல்கள் \\ விஜயலட்சுமி \\ இந்திய ஆய்வியல் துறை, மலாயாப் பல்கலைக்கழகம். \\ viijay@um.edu.my
}

\begin{abstract}
ஆய்வு சுருக்கம்
எழுத்தாளர் கே.எஸ். மணியத்தின் ஆங்கில மொழி சிறுகதைகள், நாவல்கள், நாடகங்கள் அனைத்து வகை இலக்கியப் பிரதிகளும் மலாயா/மலேசியாவின் புலம்பெயர் சமூகம் இதுவரை கடந்துவந்திருக்கும் பாதையையும் இனி செல்ல வேண்டிய இலக்கையும் அதற்கான வழிகளையும் மீளாய்வு செய்பவை. ஒவ்வொரு சமூக உறுப்பினரும் தனது மரபுவழி அடையாளங்களையும் வாழும் நாட்டின் அடையாளங்களையும் ஒருங்கே அணைத்துக் கொள்வதன்வழி பன்முகத் தன்மைகொண்ட ஒன்றைச் சமூகமாக உருவெடுக்க முடியும் எனும் பெரும் எதிர்பார்ப்பை கே.எஸ். மணியத்தின் படைப்புகள் முன்வைக்கின்றன. இதுவரையில் இவரது படைப்புக்கு கிடைத்த மிக உயரிய அங்கிகாரம் ராஜா ராவ் விருது ஆகும். இதைத் தவிர அவருடைய 'The Return' நாவலும் இதர சில சிறுகதைகளும் இடைநிலைப் பள்ளிகளில் ஆங்கில இலக்கியப் பாட நூல்களாகப் பயன்படுத்தப்பட்டதும் குறிப்பிடத்தக்க விடயமாகும். மலேசிய ஆங்கில இலக்கியச் சூழலில் முன்னோடியாகத் திகழும் கே.எஸ்.மணியத்தின் படைப்புகளில் வெளிப்படும் தனிமனித, சமூக அடையாளத் தேடல்கள்; அதை நோக்கிய பயணங்களின் சுவடுகளை அடையாளம் கண்டு விளக்க இக்கட்டுரை முனைந்துள்ளது.

கருச்சொற்கள்: கே.எஸ்.மணியம் சிறுகதைகள், கே.எஸ்.மணியம் நாவல்கள், மலேசிய ஒப்பீட்டு இலக்கியம், இந்தியர்களும் அடையாளமும்
\end{abstract}

\begin{abstract}
Almost all literary works of K.S.Maniam, regardless to genre, i.e., short stories, Novels, and Dramas, have touched the live-circle of migrant community in Malaysia. His works have discussed the milieus facing the community and the solutions for better livelihood in the foreign land for the migrants. K.S.Maniam's literary works, in particular, projects ways in which the migrant community could receive in and accept the assimilation of the local cultural essences to form a true Malaysian-based cultural infusions. For his significant contributions, the author has been honoured with the honorary award of Rajah Rao, so far. Beyond this, his monumental work, 'The Return' novel and a few short-stories have been used as literary texts in the secondary schools, as well. Unfortunately, his works have received not much appreciation among the Indian community. This article is aimed at studying and highlighting the contributions of K.S.Maniam in promoting the sense of humanity and identity in the alienated setting among the migrant community.
\end{abstract}

Keywords: K.S.Maniam Short Stories, K.S.Maniam Novels, Malaysian Comparative Literature, Indian and Indentity

\title{
முன்னுரை
}

“நான் யார்?", “எங்கிருந்து வந்தேன்?", “இனி நான் செல்லபோகும் இடம் எது?" போன்ற அடிப்படையான ஆனால் அர்த்தம் பொதிந்த இக்கேள்விகளே மனிதன் தன்னைக் குறித்த அடையாளத்தை நிர்மாணித்துக்கொள்ளவும் தான் யாரெனத் தெளிவாக மீட்டுணரவும் செய்கின்றன. இந்தக் கேள்விகளுக்குப் பதில் அளிக்கவும் சுய அடையாளத்தை உருவாக்கிக்கொள்ளவும் ஒருவர் இனம், மதம், தனது சமூக-பொருளாதார நிலை, அரசியல், அல்லது குறைந்தபட்சம் தன் குடும்ப வரலாற்றுப் பின்புலம் என பல்வேறு விடயங்களைக் கருத்தில்கொள்ள வேண்டியது அவசியமாகின்றது. அகவயத் தேடலின்; அடைதலின் அத்தனை ஏக்கமும் ஆர்வமும் புறவயமான தேடலிலும் மனித மனங்களில் தேங்கி வழிந்தபடியேதான் இ இருக்கின்றன. வெறும் பொருள்சார் தேடலாக இல்லாமல் இதுவரை வாழ்ந்த தன் மூதாதையர்களின் இருத்தலை கேள்வி எழுப்புவதோடு தன் இருப்பையும் தடுமாற்றத்துடன் மீள்பார்வை செய்பவையாக இப்புறவயத் தேடல்கள் அமைகின்றன.

கே.எஸ். மணியத்தின் படைப்புகளில் அதிகமானவை இப்புறவயத் தேடல் சார்ந்தவையாகவும் அதனுள் ஏற்படும் சிக்கல்களைத் தொடர்ந்து வட்டமிட்டபடியேவும் இருக்கின்றன. அவரது காலத்தை ஒட்டிய சக 
படைப்பாளிகள் தோட்டப்புற வாழ்வியலையும், காலனித்துவ ஆட்சியின் கொடூங்கரங்களையும், தேசப்பற்றையும் தங்களது படைப்புகளாக்கிக் கொண்டிருக்கையில் மணியத்தின் சிறுகதைகள் அடையாள நெருக்கடி (identity crisis), பன்முக கலாச்சாரவாதம் (multiculturalism), பன்மை கலாச்சாரவாதம் (cultural pluralism) என சுய, சமூக, கலாச்சார, தேசிய அடையாள நிர்மாணிப்பை மேற்கொள்வதும் கட்டுடைப்பதுமாக இருப்பது அவரை இதர பின்காலனித்துவக் காலப் படைப்பாளர்களிடமிருந்து வேறுபடுத்திக் காட்டுவதாக உள்ளது.

பின்-காலனித்துவப் படைப்பாளர்களில் மிக முக்கியமானவர்களாகக் கருதப்படும் சசல்மான் ருஷ்டி (Salman Rushdie), ஜீன் ரைஸ் (Jean Rys) போன்றவர்களின் கதைகளிலும் இம்மாதிரியான மானுடத்தின் அடையாளத் தேடல்கள் தொடர்ந்து அழுத்தமாக நிறுவப்பட்டிருப்பது காலனித்துவம் மனிதனின் 'வாழ்தல்', 'இருத்தல்' ஆகிய இரண்டுக்குமிடையே விட்டுச்சென்ற மிகப்பெரும் அடையாள தொலைதலைக் காட்டுகிறது (Hema, 2011). ஐரோப்பிய ஏகாதிபத்திய ஆக்கிரமிப்பு மனித இருப்பின் மீதான நுண்ணிய பார்வையை பல பின்-காலனித்துவ படைப்பாளர்களிடம் விட்டுச் சென்றுள்ளது. அந்த வரிசையில் கே.எஸ். மணியம் மலேசிய மண்ணுக்கு கூலித் தொழிலாளியாக வந்த ஒட்டுமொத்த தமிழர்களின் அடையாள நெருக்கடியின் சாட்சியாக நிற்கிறார்.

1916ஆம் ஆண்டு இந்திய துணைக்கண்டத்திலிருந்து ஒப்பந்தக் கூலிகளாக இந்நாட்டுக்கு புலம்பெயர்ந்த தமிழ் குடும்பத்தின், இரண்டாம் தலைமுறையைச் சேர்ந்தவர் கே.எஸ். மணியம். ஓரளவு வசதியான வாழ்வை ஏற்படுத்திக்கொள்ள, துணி சலவை செய்யும் தொழிலுடன் ரப்பர் மரம் சீவும் வேலையும் பார்த்துவந்த தந்தைக்கு எடுபிடியாகச் செல்லும் மணியம் சிறுவயதிலிருந்தே தோட்டப்புற வாழ்வையும் தோட்டத் தொழிலாளர்களையும் அறிந்திருந்தவர். அதுவரையில் இந்திய சமூகத்தினருடனே வாழ்ந்து கொண்டிருந்த மணியம் தொடக்கக் கல்வியைத் தமிழ்பள்ளியிலும், தொடர்ந்து ஆங்கிலவழி கல்வியைச் சுங்கைப்பட்டாணி இப்ராஹிம் ஆங்கிலப் பள்ளியிலும் தொடர்ந்தார். இங்கிருந்தே மணியத்தின் அடையாள நெருக்கடி, அடையாள மீட்டுணர்தலின் தேடல் தொடங்கியதாகப் புரிந்துகொள்ள முடிகின்றது. தமிழ்நாட்டிலிருந்து பிரத்தியேகமாகத் தருவிக்கப்படும் புத்தகங்களைப் பாடநூல்களாகக் கொண்டு தமிழ்நாட்டைச் சார்ந்த தமிழ்க் கல்வியையும் அதனூடே தமிழ் கலாச்சாரத்தையும் பற்றி அறியத் தொடங்கியத் தருணம் சற்றும் பரிட்சயம் இல்லாத ஆங்கிலவழிக் கல்வியும் காலனித்துவ கலாச்சாரமும் வழுக்கட்டாயமாக மணியத்தின் மூளைக்குள் புகுத்தப்பட்டது அக்காலப் பகுதியில்தான். இதனை மணியம் ‘The Return’ எனும் தன்வரலாற்று நாவலில் ரவி எனும் கதாபாத்திரத்தின் வழியாகச் சொல்கிறார்.

“என்னதான் முருகேசு வாத்தியார் தன் வாழ்நாள் அனுபவங்களையும் பல தமிழ் நூல்களையும் பயன்படுத்தி இந்துப் பையன்களுக்குப் பாடம் போதித்தாலும், மிஸ் நான்சி மிகக் கெட்டிக்காரத்தனமாக வெறும் தேவதைக் கதைகளை வைத்தே இந்து மதத்தின், சமூகத்தின் நடைமுறைகளைக் கிண்டல் செய்து புறக்கணித்து விடுவார். மேலும், அவர் இச்சிறுவர்களின் பண்பாடு, கலாச்சாரத்திற்கு முற்றிலும் முரண்பட்ட நெறிகளை அறிமுகப்படுத்திக்கொண்டே இருப்பார்." (Maniam, 1993)

என்பதாக அமையும் இந்நாவலின் பகுதி, சிறு வயது தொட்டே இப்படியான அடையாள மோதல்கள் அவருக்குள் நிகழ்ந்திருப்பதன் விழைவாகவும், சமூகத்தின் மீதான தொடர் அவதானிப்புகளும் தொடர்ந்து பன்மை கலாச்சாரவாதத்தின் மேலிருந்த ஈர்ப்பும்தான் ‘நான் யார்?’ என்கிற கேள்வியைக் கதைகளில் தொடர்ந்து அவரை கேட்க வைத்துக்கொண்டே இருந்துள்ளதாகக் காட்டுகிறது. அவரது கதைகள் காலனித்துவ ஆட்சிகால அல்லது அதற்குப் பின்பான காலவெளியில் வாழ்ந்த (புலம்பெயர்) சமூகத்தைக் காட்டுபவையாக மட்டும் இல்லாமல் இக்காலத்திற்கும் பொருந்தி நிற்பவையாக இருக்கின்றன.

\section{மலேசியாவின் வந்தேறிகள்}

கே.எஸ்.மணியத்தின் படைப்புகள் குறித்து அதிக அளவில் புலம்பெயர்வு தொடர்பான ஆய்வுகளே (Diaspora studies) மேற்கொள்ளப்பட்டு வந்துள்ள. அவரது படைப்பின் நோக்கமும் அதுவாகவே இருந்துள்ளது. இவற்றோடு மேலும், அவருடைய கதைகளில் மிக நுட்பமாக விவாதிக்கப்பட்டுள்ள தனிமனித, சமூக விழுமியங்களும் அவரது கதைகளை உயர்த்திப்பிடித்து நிற்கின்றன.

கே.எஸ். மணியத்தின் (1994) கருத்துப்படி 'புலப்பெயர்வை வெறுமனே ஒரு நாட்டைவிட்டு மற்றொரு நாட்டுக்குச் செல்லும் சாதாரணச் செயல்பாடாகப் பார்த்துவிட முடியாது. இதுவரை தான் வாழ்ந்திருந்த, தன்னோடு ஒன்றிவிட்ட குடும்பம், உறவுகள், பழகிப்போன சமூகச் சூழல், பண்பாடு என அனைத்திலிருந்தும் உணர்வுரீதியாக பெயர்த்தெடுத்து வேறொன்றோடு இணைத்துவிடும் தன்மை கொண்டாத புலப்பெயர்வு அமைகின்றது. ஒருவரது புலப்பெயர்வு அவரை மட்டுமல்லாமல் அவரின் தொடர் வம்சாவளியையே இந்த அடையாள நிர்மானிப்பைச் செய்ய நிர்பந்திக்கக் கூடியவையாக இருக்கின்றது'.

இக்கூற்றை 'Arriving' மற்றும் 'Haunting the Tiger' ஆகிய கதைகளில் இருவேறு கோணங்களில் காண முடிகின்றது. 'Arriving' கதையில், இந்தியாவைத் தனது தாய்நாடாகவும் அதையே தனது அடையாளமாகவும் காட்டிக்கொண்டிருக்கும் தனது தந்தையின் குரலை முற்றிலும் நிராகரித்துவிடும் கிருஷ்ணன் மலேசியாவையே 
தனது நாடாக உறுதியுடன் நம்புபவராக இருக்கின்றார். கூலித் தொழிலாளியாக வந்த தனது தந்தையின் பயண அனுபவங்கள், நினைவுகள், தாய்நாட்டைப் பற்றிய ஏக்கங்கள் என எதுவுமே தன்னைத் துளியும் பாதிக்காத வண்ணம் தன் இருப்பை நிலைநிறுத்திக் கொள்கிறார். கிருஷ்ணன் பிறந்தது முதல் வளர்ந்தது, படித்தது, வேலை செய்தது, திருமணம் செய்தது, குழந்தைகள் ஈன்றது, சொத்துகள் வாங்கிச் சேர்த்தது, பணி ஓய்வு பெற்றது என அனைத்தும் தன்னுடைய வீடாக (home) நினைக்கும் மலேசியாவில்தான். திவாலாகிவிடும் அபாயத்தையும் சரிகட்டி வாங்கிய வீட்டைத் தவிர்த்து அவர் மலேசியாவைச் சரீரம் தொட்டு ஆன்மா வரை ஊடிருவி நிற்கும் வீடாகவே எண்ணி வாழும் 'அப்பாவி'யாக இருக்கின்றார். இதற்கிடையில் வரும் மாட் (Mat), கிருஷ்ணனைத் திட்ட வந்தேறி (pendatang) என்ற சொல்லை வசைமொழியாகப் பயன்படுத்துகிறான். அந்தச் சொல்லுக்குள் சிக்கிக்கொள்ளும் கிருஷ்ணன் பிறகு எப்படிக் குழம்பி, மனம்நொந்து இறுதியில் அதிலிருந்து விடுதலை பெறுகிறான் என்பதாகக் கதை முடிகிறது.

இக்கதையின் நாயகனான கிருஷ்ணன் மலேசியாவிலேயே பிறந்து முக்கால்வாசி வாழ்வையும் கடந்துவிட்டவர். ஆனால், இந்நாட்டின் மண்ணின் மைந்தனாக (Son of the soil) கருத்தப்படும் மலாய்க்காரர் ஒருவர் அவரை அந்நாட்டில் 'வந்தேறி' என்று பழிப்பது மிகப்பெரும் அடையாள நெருக்கடியை அவருக்குள் விட்டுச் செல்கிறது. 'What did it mean, pendatang? Arrivals? Illegals?' என்கிற குழப்பத்தை அவருக்குள் ஏற்படுத்திவிடுகின்றது. இக்கதை எழுதப்பட்டு பத்தாண்டுகளுக்கு மேலாகிய பின்பும் 2008ஆம் ஆண்டில் இப்படியானதொரு சாடலை இந்நாட்டின் ஆளுங்கட்சித் தலைவர் ஒருவர் தேர்தல் பிரச்சாரத்தின்போது முன்வைத்ததால் பெரும்சர்ச்சைகள் ஏற்பட்டதும், அதன் நீட்சியாக இச்செய்தியை நாளிதழில் பதிவிட்ட சீனப் பத்திரிக்கை நிருபர் உள்நாட்டுப் பாதுகாப்புச் சட்டத்தின் (ISA) கீழ்க் கைதுசெய்யப்பட்டதும் குறிப்பிடத்தக்கதாகும் (Shantini \& Ganakumaran, 2009). இக்கதையில் வரும் கிருஷ்ணனின் தந்தைக்கு ‘என் தாய்நாடு' என்று சொல்லிக் கொள்ளும் 'home land' என்ற ஒன்று இருப்பதும் கிருஷ்ணனைப் போன்ற புலம்பெயர்ந்தவர்களின் சந்ததியினருக்கு 'At home and yet not home!' எனும் நிர்க்கதியான உணர்வே எஞ்சி நிற்பதையும் மிக நுட்பமாகச் சொல்லும் இக்கதையை இப்போதல்ல இன்னும் ஐம்பது ஆண்டுகள் கழித்து வாசித்தாலும்கூட நூதனமான உணர்வைக் கொடுப்பது உறுதி.

மணியத்தினுடைய கதைகளில் வரும் கதாபாத்திரங்கள் இந்நாட்டில் வாழும் சமூகங்களையும் அவர்களின் பலதரப்பட்ட இயல்புகளையும் நடுநிலையோடு முன்வைப்பதாக இருக்கின்றன. அப்பாத்திரங்களின் வாயிலாக மலேசியாவின் நுண்அரசியலையும் மணியம் மிக அழுத்தமாகவே பல கதைகளில் பதிவுசெய்துள்ளார். 'Arriving' சிறுகதையில் மாட் (Mat) எனும் கதாபாத்திரம் ஓரிரு இடங்களில் மட்டுமே வந்தாலும் அவனது பாத்திரப் படைப்பு 'Dynamic' தன்மையுடையதாக கதை முழுவதும் ஆக்கிரமிப்புச் செய்து நகர்த்துவதாக உள்ளது. மேலாதிக்க சமூகம் சிறுபான்மையினரை தங்களுக்குள் ஒருவராக சேர்த்துக் கொள்ளாமல் மேட்டிமை குணத்தோடு புறக்கணிக்கிறது என்பதை நிறுவ 'dynamic character' வகைசார்ந்த இப்பாத்திரப் படைப்பு வலுவாக அமைக்கப்பட்டுள்ளது. மறுநிலையில், 'The Third Child' எனும் சிறுகதையில் பல இடங்களில் தோன்றி மறையும் மாட் (Mat)எனும் கதாபாத்திரம் கதைக்குப் பெரிதும் முக்கியத்துவமற்ற, பணிந்து போகின்ற வகையில் 'static character' வகை சார்ந்தும் வேலு மற்றும் வசந்தி ஆகிய கதாபாத்திரங்களை மாட் மீது அதிகாரம் செலுத்தும்படியாகவும் அமைத்துள்ளார். இவ்விடம், மணியம் இந்நாட்டில் வாழும் மேலாதிக்க சமூகம் மற்றும் சிறுபான்மை/புலம்பெயர் சமூத்திற்கும் இடையில் நிகழ்ந்து கொண்டிருக்கும் சமூக இயக்கவியலை (social dynamic) கதாபாத்திர அமைப்புகளின்வழி பதிவு ெய்திருப்பதைக் காண முடிகின்றது. மேலும் இக்கதையில் வரும் 'ரிச்சர்ட' சீன சமூகத்தினரையும், ‘மைடின்' இந்தியச் முஸ்லிம் சமூகத்தினரையும் பிரதிநிதிப்பவர்களாகவும் அவர்களுக்கிடையில் ஏற்படும் அணுக்கமான உறவும் புரிந்துணர்வும் ஒருவரது தனிப்பட்ட, சமூக அடையாளத் தேடலுக்கு மத்திய9ல் 'மலேசியர்கள்' எனும் தேசிய அடையாள உருவாக்கத்தின் தேவையை முன்னிறுத்துவதாகப் புரிந்துகொள்ள முடிகிறது.

\section{தேசிய அடையாள உருவாக்கம்}

மேலும், ஒரு குறிப்பிட்ட இனக் குழுவை மண்ணின் மைந்தர்களாக பாவிக்கும் மலேசியா போன்ற நாட்டில் தேசிய அடையாளம் எவ்வாறு சாத்தியப்படும் என்ற கேள்வியையும் அதனுள் சிலந்தி வலையாகப் பின்னியிருக்கும் சிக்கல்களையும் மணியத்தின் இதர பல கதைகள் சுட்டிக் காட்டத் தவறவில்லை. இம்மண்ணிலேயே பிறந்து, வளர்ந்து, இந்நாட்டின் வளர்ச்சிக்கும் மேம்பாட்டிற்கும் உழைத்துக்கொண்டிருக்கும் இந்திய, சீன சமூகத்தினரின் மனதில் எழும் மிக அடிப்படையான கேள்வி வந்தேறிகள் (pendatang) அல்லது அதற்கு ஈடான இதர அடையாளங்களே தங்களுக்கு மிஞ்சுமா?' என்பதாகும். அடிப்படையில் இந்திய, சீன சமூகத்தினரை போலவே மலாய் இனத்தவர்களும் சில நூற்றாண்டுகளுக்கு முன்பு தங்களது பொருளாதாரத் தேவைக்காக இந்நாட்டிற்கு வந்ததது மலேசிய சரித்திரத்தில் தெரிந்தே நழுவவிடப்பட்ட உண்மையாகும். அவர்களின் பூர்வீகம் சுமத்ரா மற்றும் ஜாவா என்பதாக பல வரலாற்றாசிரியர்களும் ஆதாரம் காட்டுகின்றனர். 'Pendatang' எனும் சொல் வேறொரு 
நாட்டைப் பூர்வீகமாக கொண்டிருப்பவர்களைக் குறிக்குமெனில் இச்சமூகமும் அதற்குள் அடங்கும் (The Nut Graph, 2010). நிலைமை இப்படியிருக்க, மலேசிய அரசியலமைப்புச் சட்டம் Article 153 மிகத் தெளிவாக மலாய் சமூகத்தினரை இம்மண்ணின் மைந்தர்களாக அங்கீகரித்து அவர்களின் உரிமைகளை மட்டும் பாதுகாப்பதாக இருக்கிறது (Andrew, 2012).

சர்ச்சைக்குரியதாகக் கருத்தப்படும் இவ்விடயம் மணியத்தின் 'Arriving' கதையில் மிகப் பூடகமாகச் சொல்லப்படிருப்பது குறிப்பிடத்தக்கதாகும். இந்நாட்டைத் தனது தாய்நாடாக கருதும் ஒருவன் வந்தேறி என ஒதுக்கப்படுவதால் அவன் தொடர்ந்து தனக்கான அடையாளத்தை மட்டும் கட்டமைக்கவும் தக்கவைத்துக்கொள்ளவும் முற்படுவான் என்பதை இக்கதையின் முடிவிலிருந்து அறிய முடிகின்றது. அதேவேளை 'Haunting the Tiger' கதை, புலம்பெயர் சமூகமாக வாழ்பவர்களுக்கு தங்களது அடையாளத் தேடலுக்குரிய நுட்பமான அணுகுமுறையைச் சொல்வதாக அமைகின்றது.

மரண நாட்களை எண்ணிக்கொண்டிருக்கும் கிழவனின் எண்ணவோட்டங்களாகவும் மன அலைக்கழிப்புகளாகவும் தொடங்கும் இக்கதை சுல்கிப்லி (Zulkifli) மற்றும் முத்து ஆகிய இருவரின் வனவேட்டை அனுபவங்களைச் சொல்வதாக உருவக (Metaphor) பாணியிலேயே நகர்கிறது. தொடக்கத்தில் காட்டுப்பன்றி வேட்டைக்குத் தனியாகச் செல்லும் முத்து, பிறகு சுல்கிப்லியுடன் சேர்ந்து புலி வேட்டைக்குச் செல்கிறான். வேட்டையின்போது அவர்களுக்கிடையில் நடைபெறும் உரையாடல்கள் culture assimilation மற்றும் cultural immersion ஆகிய இருபெரும் விடயங்களை எளிமையான மொழியில் உணர்த்திக்கொண்டே தேசிய அடையாள உருவாக்கத்தின் சாத்தியங்களை வாசக மனங்களுக்குப் புரிய வைக்கின்றது. தேசிய அடையாளத்தை உருவாக்குவதில் இரர்டட வழிகள் இருப்பதை மணியம் மிகத் திடமாக நம்புகிறார் (Maniam, 1994). ஒன்று, முன்பின் அறியாத ஒரு அடையாளத்துடன் முழுவதுமாக ஒன்றிவிடுவது; மற்றொன்று சுய அடையாளத்திலிருந்து கொண்டே மற்றதையும் ஏற்றுக்கொள்வது. இக்கதையில் வரும் ஒரு பகுதியில் மணியம் பூடகமாக ஆனால் மிகத் தெளிவாக இதனைச் சொல்லிக் கடக்கிறார்.

“இதில் என்ன சிக்கலென்று எனக்குத் தெரிந்துவிட்டது. ஏதோ ஓர் அந்நிய வாடையை புலி கண்டுகொண்டது. அந்த வாடை போகும் வரை புலி வெளியே வராது," என சுல்கிப்லி கூற முத்து மிகுந்த பதற்றமடைகிறான்.

“என்ன வாடை?" எனக் கேட்கிறான்.

“மனதிலிருந்தும் உடலிலிருந்தும் வெளியாகும் வாடை. உன் உடை உன் எண்ணம் இதெல்லாம் எங்கிருந்து வந்தது? இப்படியிருந்துகொண்டு மனிதர்களை நெருங்குவதுபோல் நீ இதுனுடன் நெருங்க முடியாது. உன் மணமும் உடையும் புலி வாழும் இடத்திற்கு ஒத்திருக்க வேண்டும்".

“ஏன் ஒத்திருக்க வேண்டும்? நான் என் தந்தையின் பிடியிலிருந்து உடைபட்டு வர வேண்டும். அதுபோதும்."

“பிறகெப்படி நீ புலியாகி அதை பிடிக்க முடியும்?"

“பச்சோந்தியை மனதில் நினைத்தவாறே “நான் மாறுவேன்," என்கிறான் முத்து.

சில நூற்றாண்டுகளுக்கு முன்பு இ இம்மண்ணில் வந்த தனது மூதாதையர்கள் இநந்ாட்டின் கலாச்சாரத்துடன் ஒன்றியவர்கள் எனும் அடிப்படையில் அதே உத்தியை முத்துவிற்கும் சுல்கிப்லி சொல்கிறான். புலியாக மாறுவது என்பதை இந்நாட்டின் பண்பாட்டு கலாச்சாரத்துடன் ஒன்றிவிடும் செயலாக நாம் புரிந்துகொள்ள முடிகிறது. ஆனால், முத்து கடைசிவரை அவ்வாறு செய்யாமல் தன்னை பச்சோந்தியாகவே மற்றிக்கொள்ள முயற்சிக்கிறான் என்பதாகக் காட்டுவது அவன் தனது மரபுகளை தக்கவைத்துக் கொண்டு புதியதையும் ஏற்க முற்படுகிறான் என்பதாகப் புரிந்து கொள்ளலாம்.

முத்துவைப் போலவே பலர் தங்களது பண்பாடு, கலாச்சாரம், மரபுகள் என தங்களுக்கே உரிய அடையாளங்களுடன் தாங்கள் இந்நாட்டில் ஓர் அங்கமாக அறியப்பட்த் தொடர்ந்து முயற்சித்துக் கொண்டே இருப்பதை இன்றும் காண முடிகிறது. மூவின மக்களையும் ஒன்றுப்பட்ட ஒற்றைச் சமூகமாக மாற்ற இந்நாட்டு அரசாங்கம் மேற்கொண்ட Wawasan 2020, Sekolah Wawasan, 1Malaysia போன்ற பற்பல திட்டங்கள் இந்திய, சீன சமூகத்தினரிடையே பெரிய அளவில் ஆதரவைப் பெறாமல் போனதற்கும் இவ்வகை மரபுசார்ந்த அடையாளக் கட்டமைப்புகளும் ஆதிக்கச் சமூகத்தின் கெடுபிடிகளும் அடிப்படைக் காரணமாக இருப்பதை இவ்விடம் நினைவு கோரலாம்.

புலம்பெயர்ந்த சமூகத்தினருக்கு எப்போதுமே அந்நாட்டின் பண்பாட்டு, கலாச்சார, மரபுகளுடன் ஓரளவு சமரசம் செய்துகொண்டு, அம்மண்ணை முழுவதும் ஏற்கவும் முன்னோர்களின் தாய்நாட்டு மோகத்திலிருந்து 
விடுபடுவும் உள்ளழுத்தங்கள் ஏற்படுவது நிதர்சனம். புலம்பெயர்ந்தவர்கள் தாங்கள் வாழ்கின்ற நாட்டின் தொடர்ச்சியான மாற்றங்களை உள்வாங்கிக்கொண்டு அதனூடே தங்களது அடையாளங்களையும் வழுவாகப் பிடித்துக் கொள்பவர்களைக் காலத்திற்கேற்ப வாழும் new diasporic man என்று மணியம் வர்ணிக்கிறார்.

'Haunting the Tiger' சிறுகதை முழுவதும் முத்து எந்த வழியையும் தேர்ந்தெடுக்க முடியாமல் தடுமாறும் நிலைத்தன்மையற்ற ஆன்மாவாக அலைக்கழிவதாக காட்டப்படுகிறது. ஒரு நல்ல சிறுகதை தானாக வலிந்து சென்று எந்தக் கருத்தையும் முடிவையும் சொல்வதில்லை. அவை அதற்கான வேலையை வாசக மனங்களிடமே விட்டுச் செல்கின்றன. அவ்வகையில் இக்கதையை வாசித்து முடிக்கும் தருணம் வாசகன் குறைந்தபட்சம் முத்துவினுடைய அத்தனை அலைக்கழிப்புகளையும் தன்னுள் உணரக்கூடும். அப்படி நடக்குமாயின் அதுவே மணியத்தின் வெற்றியாகவும் இருக்கும்.

ஏறக்குறைய மூன்று அல்லது நான்காவது தலைமுறையைத் தொட்டுவிட்ட மலேசியத் தமிழர்களும் சீனர்களும் இந்நாட்டின் ஓர் அங்கமாக தங்களை இணைத்துக்கொள்ள முடியாமல் தங்களது தனித்த அடையாளத்தையும், அங்கீகாரத்தையும் மட்டுமே நிறுவ முயலுவார்களெனில் அது இன ரீதியான பாகுபாட்டைத் தொடர்ந்துவரும் சந்ததியினரின் மனதில் ஆழ வேரூன்ற செய்துவிடும் எனும் பதைபதைப்பையும் மணியத்தின் கதைகளில் காண முடிகின்றது. In A Far Country நாவலில் வரும் Lee Chin எனும் சீன கதாபாத்திரமும் Pelanduk எனும் சிறுகதையில் வரும் ஆரோக்கியன் எனும் கதாபாத்திரமும் தீவிரமான சுய அடையாள நிர்மானிப்புகள் எவ்வாறு அவர்களைக் குரூரமானவர்களாக மாற்றுகின்றது என்பதைச் சித்திரிக்கின்றன.

Pelanduk சிறுகதையில் வரும் ஆரோக்கியன் தொடக்கத்திலிருந்தே மிக மெல்லிய உணர்வாளனாகச் சித்திரிக்கப்படுகிறான். ஆனால், அவனிடம் தொடர்ந்து சொல்லப்படும் சீதை மான் மேல் கொண்ட மோகமும், அதைபிடிக்கச் சென்ற ராமன் அதுவரையில் விரட்டியது மானல்ல மாறாக மானாக மாறிவிட்ட மாரீசன் என்பதை அம்பு எய்திய பிறகு கண்டுகொள்ளும் ராமாயண (புராண) கதையில் முழுவதும் தன்னை அர்ப்பணிக்கிறான். அதே போன்றதொரு மானைத் தங்கள் குடியிருப்புப் பகுதியில் இருப்பதாகச் சொல்லி அவனை வேட்டைக்கு வலுக்கட்டாயமாக அனுப்பும் சூழலில் உடன் சென்ற பாண்டியன் என்பவனைக் கொன்று விடுகிறான். ஊரே பாண்டியன் திரும்ப வராதது குறித்து கேட்கையில் “நான் மானைக் கொன்றுவிட்டேன். ஆனால், மாரீசன் போலவே அதுவும் மனிதனாகி விட்டது," என்கிறான். இக்கதையில் சொல்லப்படும் புராணம் இந்திய சமூகத்தின் தனிப்பட்ட அடையாளத்தையும், பாண்டியன் என்ற கதாபாத்திரம் வேறொரு சமூக அடையாளத்தையும், ஆரோக்கியன் கொலை செய்யும் சம்பவம் ஒரு சமூகம் எப்படிக் குருட்டுத்தனமாக சில அடையாளங்களைப் பின்பற்றுகிறது என்பதையும் உருவக பாணியில் சொல்லிச் செல்கிறது (Shantini, \& Ganakumaran, 2009). இச்சிறுகதையில் வரும் ஆரோக்கியன் போலவே பலரும் தங்கள் அடையாளங்கள் இன்னதென குருட்டுதனமாக நம்பிக்கொண்டு அவற்றைத் தற்கால சூழலோடு பொருத்திக்கொள்ள முடியாத நிலையில் முரண்செயல்பாட்டிற்குத் தள்ளப்படுகிறார்கள்.

தொடர்ந்து, 'In A Far Country' நாவலில் வரும் லீ சின் தனது புல்லாங்குழலை மலேசியாவில் விளைந்த மூங்கிலைக் கொண்டு தயாரிப்பதால் அதன் புனிதமும் அசல் தன்மையும் கெட்டுவிடுவதாக உணர்கிறான். தொடர்ந்து, புல்லாங்குழலை கவனமாகப் பாதுகாப்பதும், சீனர்களின் தொல்பொருட்களை மட்டும் தேடி சேகரிப்பவனாகவும் தன்னை ஒரு வட்டத்துக்குள் நிறுத்திக் கொள்கிறான். இவ்வாறு செய்வதன்மூலம் தன் (இனத்தின்) அடையாளங்களை மீட்டெடுப்பதாகவும் கட்டிக்காப்பதாகவும் எண்ணி தன்னை ஒரு கலாச்சாரக் காவலானாக மாற்றிக் கொள்கிறான். "இந்த நாட்டில் விளைந்த மூங்கிலின் மூலம் இதுபோன்றதொரு மூங்கிலைச் செய்ய முடியாது. அப்படி செய்தாலும் அது வலுவற்று, காற்றழுத்தம் குன்றி இருக்கும்," எனக்கூறும் லீ சின் கதாபாத்திரத்தைக் கொண்டு புலம்பெயர்ந்து வாழ்பவர்கள் தங்களது அடையாளங்களை மீட்டெடுக்க நடப்பு வாழ்வுக்கு முரணாக இயங்குகிறார்கள் என்பதை புரிந்துகொள்ள முடிகின்றது. லீ சின் தனது இன, கலாச்சார அடையாளத்தை மீட்பதில் காட்டும் முரட்டுத்தனுமும் பிடிவாதமும் இறுதியில் அன்றாட வாழ்வுடன் ஒத்துப்போக முடியாமல் மனப்பிரள்வு நிலையை எதிர்கொண்டு சற்றும் எதிர்பாராத நிலையில் மரணிக்கச் செய்கிறது.

இந்நாவல் புலம்பெயர் நாடுகளில் மரபுவழி இன, கலாச்சார அடையாளத்தை மீட்டெடுப்பதில் உண்டாகும் சிக்கலை குற்றச்சாட்டாக வைக்காமல் பன்முக கலாச்சாரத்தை (Multiculturalism) ஏற்க தவறும் ஒரு சமூகம் எப்படி நிலைத்தன்மையற்று உழலும் என்பதை கோடிகாட்டுவதாக உள்ளது. மேலும் லீ சின்னினுடைய செயல்பாடுகள், ஒரு குறிப்பிட்ட அடையாள மீட்டெடுப்பு என்பது மற்றவர்களின் அடையாளங்களை தாழ்வாக பார்க்கும் வெறித்தன்மைக்கு இட்டுச்செல்லும் என்றும் முரண் செயல்பாட்டை விளைவிக்கும் என்பதையும் உணர்த்துகின்றன. இந்நாவலில் அமையப்பெற்றுள்ள மற்றொரு கதாபாத்திரம் லீ சின்னுக்கு முற்றிலும் எதிர்மறையாகச் செயல்படுவதாக அமைத்திருப்பது கதையின் நகர்ச்சியில் மிகுந்த ஆர்வத்தை ஊட்டுவதாக இருக்கிறது. லீ சின் போல் அல்லாமல், ராஜன் எதையுமே ஏற்காமலும் எந்தவோர் அடையாளத் தேடலுமின்றித் தொடர்ந்து பணத்தை மட்டும் சம்பாதித்துக் குவிப்பதன்வழி தன்னை இ இந்நாட்டின் ஓர் அங்கமாக்கிக்கொள்ள முயலுகிறான். 
இம்மாதிரியான அணுகுமுறை சரியானதா எனக் கேள்வி எழுப்பும் வகையில் சுல்கிப்லி மற்றும் ராஜன் ஆகியோருக்கு இடையிலான உரையாடல்கள் அமைகின்றன.

“என்னால் உங்களைப் போன்றவர்களைப் புரிந்துகொள்ள முடியவில்லை. நீ இந்த மண்ணை அவமதித்துக் கொண்டே இருக்கிறாய்," என்கிறான் சுல்கிப்லி.

“என்ன அவமதிப்பு?"

“இந்த மண்ணை, துணிகளை விற்பதுபோல் கூறுபோட்டு விற்கிறாய்."

“நீ ஏன் அதைக் குறை சொல்கிறாய்? நான் என் நிலத்தை விற்கவில்லை. என்னுடைய நிலம் என்று எதுவும் இல்லை," என்று ராஜன் தனது நிலைப்பாட்டை முன் வைக்கிறான்.

“நீ இந்த நாட்டை முழுமையாக புரிந்துகொண்டால் உனக்கென்று ஒரு நிலம் இருப்பதை உணருவாய்".

“நான் ஏற்கெனவே புரிந்துகொண்டேன். உலகில் உள்ள மற்ற நாடுகளைப் போலத்தான் இதுவும்," என்று தனது 'belong to nowhere' எனும் பிடிமானத்தை இன்னும் ஆழமாகச் சொல்கிறான்.

இந்நாவலில் வரும் இவ்விரு கதாபாத்திரங்களும் பன்முக கலாச்சாரங்கள் குவிந்திருக்கும் மலேசியா போன்ற நாட்டுக்கு முற்றிலும் ஒவ்வாத தன்மையைக் கொண்டதாகவே இருக்கின்றது. ஒரே அடையாளத்தை மட்டும் நிறுவ முயலுவது எப்படி இன, கலாச்சார வெறியைத் தூண்டும் அபாயம் கொண்டதோ அதேபோல அனைத்தையும் புறக்கணிக்கும் போக்கு அருவருக்கத்தக்கதாகக் காட்டப்படுகின்றது. உலகின் பல நாடுகளில் இனக்கலவரங்கள் நடப்பதையும், மொரீசியஸ், தாய்லாந்து போன்ற நாடுகளில் வாழும் தமிழர்கள் தங்களது அடையாளங்களைத் தொலைத்துவிட்டிருப்பதையும் இவ்விரும் முரண் கதாபாத்திரங்களினூடாகத் தொடர்புபடுத்திப் பார்க்க முடிகின்றது.

\section{முடிவுரை}

கே.எஸ். மணியத்தின் சிறுகதைகள், நாவல்கள், நாடகங்கள் என அனைத்தும் மலாயா/மலேசியாவின் புலம்பெயர் சமூகம் இதுவரை கடந்து வந்திருக்கும் பாதையையும் இனிச் செல்ல வேண்டிய இலக்கையும் அதற்கான வழிகளையும் அலுக்காமல் சொல்லிக் கொண்டே இருக்கின்றன. அனைத்துச் சமூக உறுப்பினர்களும் தங்களுடைய மரபுவழி அடையாளங்களையும் வாழும் நாட்டின் அடையாளங்களையும் ஒருங்கே அணைத்துக் கொள்வதன்வழி பன்முகத் தன்மைகொண்ட ஒன்றைச் சமூகமாக உருவெடுக்க முடியும் எனும் பெரும் எதிர்பார்ப்பை கே.எஸ். மணியத்தின் படைப்புகள் சுமந்து நிற்கின்றன. 62 ஆண்டுகால சுதந்திர மலேசியாவில் இன்றைய தேசிய அடையாளத்தை நோக்கிய நகர்ச்சி நிச்சயம் மீளாய்வுக்கு செய்யப்படவேண்டிய கட்டாயத்தையும் இவருடைய கதைகளின்வழி உணர முடிகின்றது. தென்னாசியாவின் புலப்பெயர்வு (South Asian diaspora) வரலாற்றுப் பதிவுகளில் இன்றியமையா இடம்பிடித்துள்ள கே.எஸ்.மணியத்தின் அடையாளங்களை தேடிச் சென்றபோது மிஞ்சியது ஏற்கமுடியாத ஏமாற்றங்கள் மட்டுமே. இதுவரையில் இவரது படைப்புக்கு கிடைத்த மிக உயரிய அங்கிகாரமாக Raja Rao Award இருக்கின்றது. இது அவரின் ஒட்டுமொத்தப் படைப்புக்கும் கிடைத்த ஒற்றை விருதாகும். இதைத் தவிர அவருடைய 'The Return' நாவலும் இதர சில சிறுகதைகளும் இடைநிலைப் பள்ளிகளில் ஆங்கில இலக்கியப் பாட நூல்களாகப் பயன்படுத்தப்பட்டதும் குறிப்பிடத்தக்க விடயமாகும்.

புலம்பெயர் சமூகமாத்தினரது வாழ்வின் அபத்தங்களையும் குறைபாடுகளையும், இன்னும் பல சர்ச்சைக்குறிய விடயங்களையும் தனது படைப்பாளுமையின்வழி மிக நுட்பமாக படைத்திருக்கும் கே.எஸ். மணியம் இன்றளவிலும் அவரின் படைப்பிற்குரிய அங்கீகாரத்துடன் அடையாளப்படுத்தப்படாமல் இருப்பது இந்நாட்டினுடைய தேசிய அடையாள உருவாக்கத்தின் வறட்சியையே காட்டுகிறது. தனிமனித, தேசிய அடையாள உருவாக்கத்தின் தேக்கத்தை எல்லா இனத்தவர்களும் படித்துணரும் வகையில் அமையப்பெற்றிருக்கும் இவரது படைப்புகள் தேசிய இலக்கிய அடையாளத்தைப் பெற முடியாமல் வெறுமனே ஒரு குறிப்பிட்ட சமூகத்தின் இலக்கியமாக எஞ்சி நிற்பது மிகுந்த வருத்தத்திற்குரியதாகும். இதனையே 'to be Indian in a non-Indian world' எனும் வாசகத்தின் பொருளாகப் புரிந்துகொள்ள முடிகின்றது (qtd. Peter Wicks, பக்.10). இந்நாட்டின் ஆதிக்க சமூகம்தான் இப்படியானதொரு கொள்கையினைக் கொண்டிருக்கிறது என்றால் இந்திய சமூகமும் மணியத்தைக் கொண்டாடாமல் இருப்பதை எவ்விடம் சொல்லி நொந்துகொள்வது?

\section{மேற்கோள்கள்}

Andrew, H. (2012). The Constitution of Malaysia: A Contextual Analysis. Oregon: Hard Publishing. 
மரபுவழி அடையாளம்-விஜயலட்சுமி

Hema, T.A. \& Madhavan Nair. (2011). Crisis of Identity in Selected Works of Jean Rhys, Salman Rushdie and K.S. Maniam. Kuala Lumpur: University of Malaya.

Maniam, K.S. (1993). In A Far Country. London: Skoob Books.

Maniam, K.S. (1993). The Return. London: Skoob Books.

Maniam, K.S. (1994). In A Search of A Centre. Doi: http://www.ucalgary.ca/uofc/eduweb/engl392/maniam-cent.html Maniam, K.S. (1996). Faced Out: Six Stories. Selangor: Maya Press.

Maniam, K.S. (1996). Hunting the Tiger: Contemporary Stories from Malaysia. London: Skoob Books.

Nut Graph. (2010). Found in Malaysia. Selangor: ZI Publications.

Peter Wicks. Malaysia as a myth in KS Maniam's In a Far Country. Doi: https://eprints.usq.edu.au/732/1/Wicks_Maniam1.pdf

Sarup, M. (1996). Identity, Culture and the Postmodern World. Georgia: The University of Georgia Press.

Schwartz, S.T., \& Marilyn, J.M. (2006). The role of identity in Acculturation Among Immigrant People: Theoretical Propositions, Empirical Questions and Applied Recommendations. Basel: Karger.

Shantini, P., \& Ganakumaran, S. (2009). Through the Coils of Memory and Ethnicity: The Malaysian Indian Journal of Nationwood. European Journals of Social Sciences. Vol 7(3).

Zedeck Siew. (2012). Having to Belong Everywhere. [Interview Transcript]. Retrieved from http://penangmonthly.com/having-to-belong-everywhere/ 